\title{
Growth of quantum dot coated core-shell anisotropic nanowires for improved thermal and electronic transport
}

Cite as: Appl. Phys. Lett. 114, 243104 (2019); https://doi.org/10.1063/1.5100891

Submitted: 22 April 2019 . Accepted: 28 May 2019 . Published Online: 17 June 2019

Bo Hou (D), Su-Ho Jung, Jingchao Zhang (D), Yang Hong, Byung-Sung Kim, Jung Inn Sohn (D), Eun Kyung Lee, Byoung Lyong Choi, Dongmok Whang (D), SeungNam Cha $\mathbb{D}^{\mathrm{D}}$, and Jong Min Kim
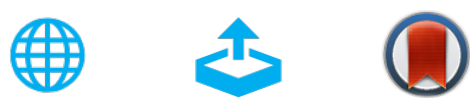

View Online

Export Citation

\section{ARTICLES YOU MAY BE INTERESTED IN}

High performance transistors and photodetectors based on self-catalyzed zinc-blende InP nanowires

Applied Physics Letters 114, 243106 (2019); https://doi.org/10.1063/1.5094647

Measurement of ion mobility based on a reversible migration process in solids Applied Physics Letters 114, 243901 (2019); https://doi.org/10.1063/1.5094204

Infrared luminescence from $\mathrm{N}$-polar InN quantum dots and thin films grown by metal organic chemical vapor deposition

Applied Physics Letters 114, 241103 (2019); https://doi.org/10.1063/1.5109734

Lock-in Amplifiers Find out more today

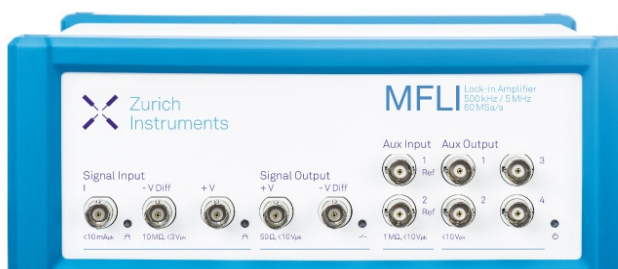

Zurich Instruments 


\title{
Growth of quantum dot coated core-shell anisotropic nanowires for improved thermal and electronic transport
}

Cite as: Appl. Phys. Lett. 114, 243104 (2019); doi: 10.1063/1.5100891

Submitted: 22 April 2019 - Accepted: 28 May 2019 •

Published Online: 17 June 2019

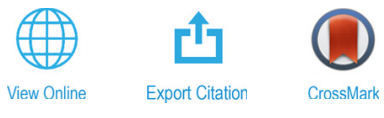

Bo Hou, ,a) (D) Su-Ho Jung, ${ }^{2, a)}$ Jingchao Zhang, ${ }^{3}$ (D) Yang Hong, ${ }^{4}$ Byung-Sung Kim, Jung Inn Sohn, ${ }^{5}$ Eun Kyung Lee, ${ }^{6}$ Byoung Lyong Choi, ${ }^{6}$ Dongmok Whang, ${ }^{2,7, b)}$ (D) SeungNam Cha, ${ }^{8, b)}$ (D) and Jong Min Kim ${ }^{9}$

\author{
AFFILIATIONS \\ 'Department of Engineering Science, University of Oxford, OX1 3PJ Oxford, United Kingdom \\ ${ }^{2}$ SKKU Advanced Institute of Nanotechnology, Sungkyunkwan University, Suwon 440-746, South Korea \\ ${ }^{3}$ Holland Computing Centre, University of Nebraska-Lincoln, Lincoln, Nebraska 68588, USA \\ ${ }^{4}$ Department of Chemistry, University of Nebraska-Lincoln, Lincoln, Nebraska 68588, USA \\ ${ }^{5}$ Division of Physics and Semiconductor Science, Dongguk University-Seoul, Seoul 100-715, South Korea \\ ${ }^{6}$ Samsung Advanced Institute of Technology, D\&S Research Centre, Samsung Electronics, Youngtong-gu, Suwon-si, \\ Gyeonggi-do 443-803, South Korea \\ ${ }^{7}$ School of Advanced Materials Science and Engineering, Sungkyunkwan University, Suwon 440-746, South Korea \\ ${ }^{8}$ Department of Physics, Sungkyunkwan University, Suwon 16419, South Korea \\ ${ }^{9}$ Department of Engineering, University of Cambridge, CB3 OFA, Cambridge, United Kingdom

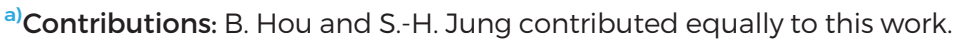 \\ b) Authors to whom correspondence should be addressed: dwhang@skku.edu and chasn@skku.edu
}

\begin{abstract}
Anisotropic nanowires are promising candidates for electronic thermal management due to their unique electrical and thermal properties. However, eco-friendly solution-processed nanomaterials with an elaborate morphology and microstructure for modulating thermal and charge transfer are still a considerable challenge. Herein, we present a simple but effective approach for synthesizing pseudo core-shell nanowires through quantum dot (QD)-like nanostructure coating ( $p-\mathrm{NW} @ \mathrm{QD})$ to generate exceptional electron-phonon transport properties. With the assistance of diphenyl ether as a coordination solvent, high crystallinity lead sulfide NWs can be fabricated with a large aspect ratio together with uniform QD coating. This $p$-NW@QD exhibits high electronic mobility $\left(30.65 \mathrm{~cm}^{2} / \mathrm{Vs}\right)$ as well as a diameter independent low thermal conductivity $(1.53 \pm 1 \mathrm{~W} / \mathrm{m} \mathrm{K})$. Direct charge/heat carrier flow measurements and computational simulations demonstrate that the unusual electrical and thermal transport phenomenon is strongly dependent on the fast charge transport through the QD shell, and a slow phonon migration across the Umklapp process dominated NW cores. These findings indicate a significant step toward colloidal synthesis nanostructures for future high-performance nanoelectronics and thermal energy devices.
\end{abstract}

Published under license by AIP Publishing. https://doi.org/10.1063/1.5100891

Thermal transport in materials is drawing persistent attention because of intriguing fundamental thermal physics in various dimensions, as well as the growing importance of heat management in electronic devices and electricity base. ${ }^{1,2}$ For instance, thermal transport can create an uneven distribution of charge carriers in semiconductors (e.g., thermoelectric effect), which could provide a global sustainable energy solution by generating electricity from waste heat. ${ }^{1,3,4}$ The fundamental principle underlying the thermal transport is the flow of electrons and phonons that carry heat and charge under a thermal gradient. ${ }^{3}$ In semiconductors, this dynamic process has been theoretically rationalized as $Z T=\sigma S^{2} T /\left(\kappa_{e l}+\kappa_{\text {lat }}\right)$ (i.e., Peltier-Seebeck Effect), where $Z T$ is the dimensionless figure of merit, $\sigma$ is the electrical conductivity, $T$ is the temperature, $S$ is the Seebeck coefficient, and $\kappa_{\text {lat }}$ and $\kappa_{e l}$ are the lattice and electronic thermal conductivities $(\kappa$ $\left.=\kappa_{e l}+\kappa_{\text {lat }}\right){ }^{3}$. Realizing a low thermal conductivity but high electrical conductivity is the figure-of-merit for achieving a temperature 
gradient in a conductive substance, which is critical for electronically thermal energy management, such as thermoelectric generators (TE). ${ }^{5,6}$ Unfortunately, $S, \sigma$, and $\kappa$ are interdependent variables, and thus, they cannot merely be tailored.

In low-dimensional nanostructures, the anisotropic spatially confined density-of-states (DOS) can provide a solution to alter one of the interrelated "Peltier-Seebeck Effect" parameters without affecting the others [Fig. 1(a)]. Recently, various approaches have been explored on the development of nanoscale structures for controlling electron-phonon interactions and transports including Fermi liquid, insulator, alloys, ${ }^{9}$ or doped semiconductors. ${ }^{10}$ In theory, the carrier transport (including charge and heat) can be controlled through dimension or compositional-lattice engineering. ${ }^{711}$ However, in order to generate a $Z T$ value that is large enough (e.g., above 3 ) to compete with conventional power generators, ${ }^{12}$ the diameter of the nanostructures, such as nanowires (NWs), ${ }^{13-16}$ needs to be extremely small (e.g., below $10 \mathrm{~nm}$ ), which challenges the limitation of the state-ofthe-art nanofabrication. ${ }^{14}$

Nevertheless, the seminal studies in Si or Ge NWs introduced a "surface phonon scattering" (SPS) strategy via NW surface modification, which showed significant improvement in TE performance. ${ }^{11,15,17}$ SPS has shown promise in altering the electrical and thermal transport, but this controllability can be significantly enhanced if fundamental differences between semiconductors such as bandgaps and exciton binding energies could be considered. ${ }^{2,6,12,14}$ For example, the exciton Bohr radius of lead sulfide $(\mathrm{PbS})$ is three times

(a)

(b)
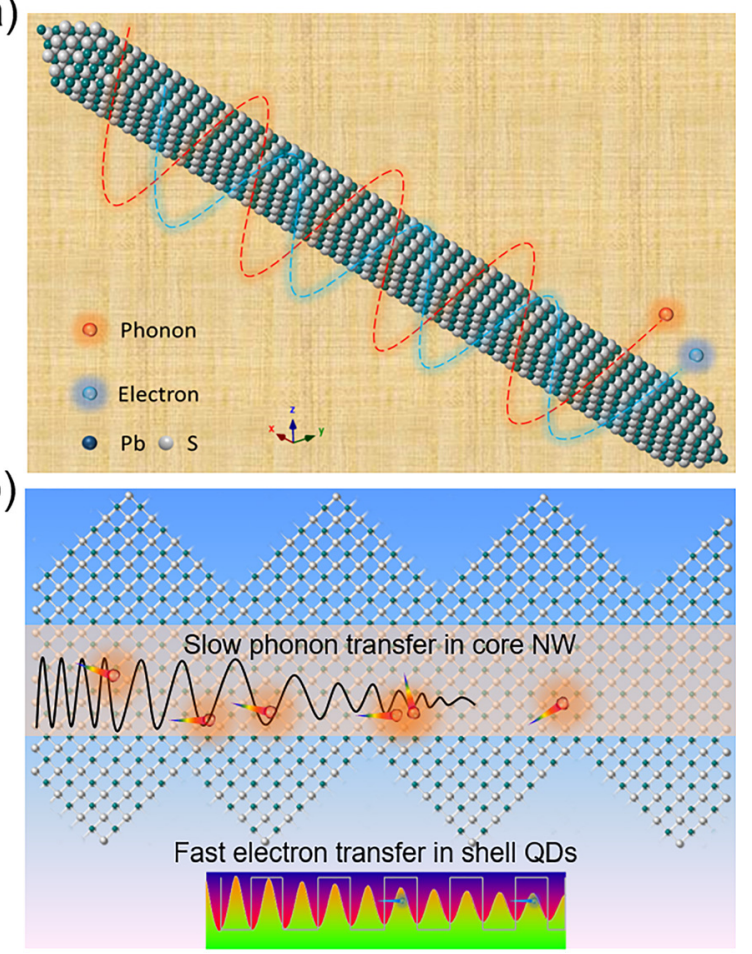

FIG. 1. (a) A schematic description of electronic and phonon transport in $\mathrm{PbS}$ NWs. (b) Cross-section schematic of the as-proposed p-NW@QD shows the "thermoelectric rectification" mechanism that enables slow phonon propagation within the NW "core" and fast electron transfer through the QD "shell." larger than that of $\mathrm{Si}$, and $\mathrm{PbS}$ has shown a strong quantumconfinement effect in manipulating charge-mobility in various optoelectronic and photonic devices. ${ }^{18-20}$ Therefore, in addition to SPS, an electronic quantum-confinement can independently influence and contribute to tuning the thermal transport (i.e., TE power factor) if we modify the NW surface into a spatially confined structure. . $7,14,17,21^{-}$

Herein, we present an approach for synthesizing pseudo coreshell nanowires with a quantum dotlike nanostructure coating ( $p$-NW@QD) through a solvent-mediated approach. With the assistance of a coordinating reagent diphenyl ether (DER), NWs not only grow along the $\langle 112\rangle$ direction but also can be uniformly coated with highly crystalline QDs. We further fabricate field-effect transistors (FETs) and a microfabricated suspended heating device to directly probe charge/heat flow dynamics within a single $\mathrm{PbS} p$ NW@QD. Based on theoretical simulations and systematic microscopy analysis, we determine that the anomalous low thermal conductivity and high charge carrier mobility originate from $p$ NW@QD, efficiently regulating the charge and phonon transport via QD shells and NW cores, respectively.

To facilitate the anisotropic crystal growth, we combined the oriented attachment methodology with a solvent-mediated crystalgrowth approach to fabricate the $p$-NW@QD core-shell nanostructure. As illustrated in Figs. 2(a)-2(c), we found that DER can play an important role in tuning the surface morphology besides its conventional role as a precursor solvent. ${ }^{22,23}$ As shown in Fig. 2(a), the morphology of the as-prepared NWs was dramatically changed when the coordinating-solvent DER was introduced. As indexed in the selectedarea electron diffraction (SAED) patterns and powder X-ray

(a)
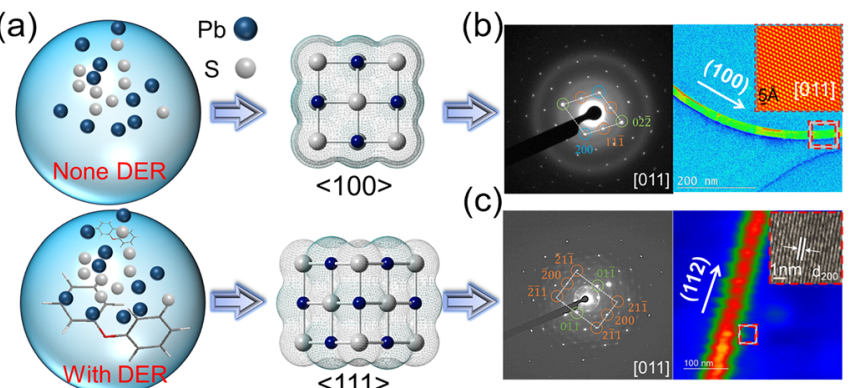

(d)

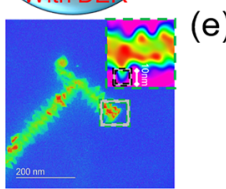

(g)
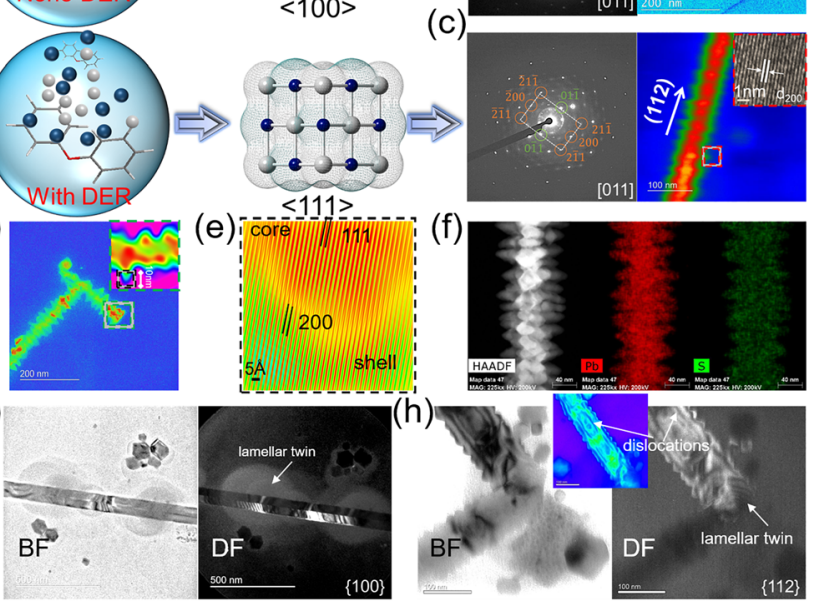

FIG. 2. (a) Schematic illustration of the synthesis process of PbS p-NW@QD coreshell structures. (b)-(e) SAED, ARM, HAADF-STEM, and HRTEM images of the as-prepared PbS NWs and p-NW@QDs in the contrast color scale. The lattice spacings of $\{200\}$ and $\{111\}$ planes were $3.0 \pm 0.1 \AA$ and $3.4 \pm 0.2 \AA$, respectively. (f) HAADF-STEM elemental mapping images of $p-N W @ Q D s .(g)$ and $(\mathrm{h})$ BF and $\mathrm{DF}$ images highlight the dislocations and lamellar twin defects in greyscale and contrast color scales (inset). 
diffraction (XRD) patterns (Fig. S1), the addition of DER in the NW growth media [1-octadecene (ODE):DER $=10: 1 / \mathrm{v}: \mathrm{v}$ ] can effectively adjust the growth direction of NWs from $\langle 100\rangle$ [Fig. 2(b)] to $\langle 112\rangle$ [Fig. 2(c)]. DER has higher nucleophilic nature compared to ODE. Therefore, it can be anticipated that DER would be bound to the $\mathrm{Pb}$ ions of the growing nanocrystals, similar to the other amines (i.e., oleylamine, trioctylamine, hexadecylamine, etc.) to control the morphology and growth direction. ${ }^{22,23}$ The as-prepared NWs and $p$ NW@QDs exhibited a high degree of crystallinity [insets of Figs. 2(b), 2(c), and S2) with diameters between $50 \mathrm{~nm}$ and $500 \mathrm{~nm}$ and lengths up to $10 \mathrm{um}$. As shown in Fig. 2(d), the QD-like nanograins (dimension 5-15 nm) formed spatially confined nanostructures [inset of Figs. 2(d) and S3] and uniformly distributed on the surface of NWs. Moreover, as shown in the false-color bright-field (BF) microscopy images [Figs. 2(d) and 2(e)], we observed an enrichment of structuraldisorder in $\langle 112\rangle$ oriented $p$-NW@QDs. As further highlighted in the atomic-resolution microscopy (ARM) aberration-corrected high-angle annular dark-field (HAADF-STEM) images, moiré fringe defects from the lattice mismatch $\{200$ and 111$\}$ and twinnings can be readily resolved at the $\langle 112\rangle$ oriented NW/QD core and shell interface [Fig. $2(\mathrm{e})] .^{22}$ The composition of the as-prepared p-NW@QDs was determined through X-ray photoelectron spectroscopy (Fig. S4) and HAADF-STEM elemental-mapping [Fig. 2(f)], which showed that a homogeneous $\mathrm{Pb}-\mathrm{S}$ distribution excluded the possibility of the presence of localized-sediments. ${ }^{9,24,25} \mathrm{We}$ further probed the structural difference between $\langle 100\rangle$ and $\langle 112\rangle$ oriented NWs through direct-beam $\mathrm{BF}$ and diffracted-beam dark-field (DF) imaging. As displayed in Fig. 2(g), only twinning dislocations and lamellar twin boundaries can be resolved in $\langle 100\rangle$ oriented NWs (Fig. S5). On the other hand, twinning, dislocations, and stacking fault bend contours can be identified in the thickness and stacking-fault fringes among the $\langle 112\rangle$ preferred p-NW@QDs [Figs. 2(h) and S6]. The large aspect ratio $(\sim 100)$, disordered lattice structure, and quantum-confined surface shell are expected advantages to give rise to exceptional thermal and charge transport properties within this p-NW@QD core-shell structure.

To gain further understandings on the dynamical thermal transport in PbS NWs under a thermal gradient, we carried out systematic density functional perturbation theory (DFPT) and molecular dynamics (MD) thermal transport simulation. As shown in Fig. 3(a), the strong anharmonicity of $\mathrm{PbS}$ is reflected in the dominated acoustic phonons from its phonon density of state (PDOS) as calculated from the DFPT. ${ }^{26,27}$ This strong anharmonic characteristic leads to large Grüneisen parameters and large phonon scatterings which were determined to be the main reason for the intrinsic low lattice thermal conductivity in $\mathrm{PbS}$ crystals (e.g., $2.5 \mathrm{~W} / \mathrm{mK}$ at $300 \mathrm{~K}$ ). ${ }^{26,27}$ Atomic configurations of the $\mathrm{PbS}$ system and the steady-state nonequilibrium molecular dynamics (NEMD) method setup are illustrated in Fig. 3(b) and the supplementary material. ${ }^{28}$ After the NEMD calculation, a steady-state temperature gradient was built along the heat flux direction [Fig. 3(c)]. ${ }^{29,30}$ As shown in Fig. 3(d), within the temperature range of $200 \mathrm{~K}-400 \mathrm{~K}$, the phonon-free-path threshold value can be estimated to be $9.6 \mathrm{~nm}$ which is close to previous predictions based on first-principles calculations. ${ }^{26}$ As shown in Fig. 3(e), when two incoming phonons (vector $k_{1}$ and $k_{2}$ ) scatter inside of the first Brillouin zone, the derived phonon $k_{3}^{\prime}$ may constrain inside or outside via the normal-scattering-process (N-process) and the Umklapp-process (U-process). ${ }^{31}$ The U-process suppresses the thermal conductivity which was reflected through exponential $\kappa$ decay as a function of temperature (a)

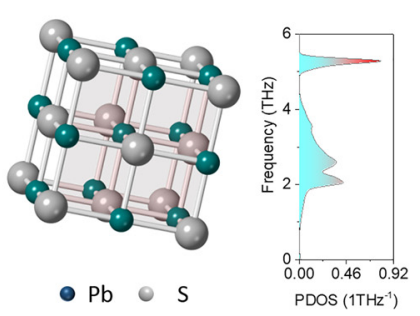

(c)

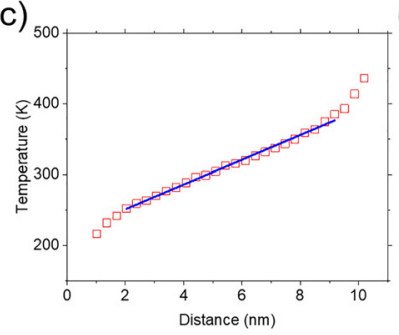

(d)
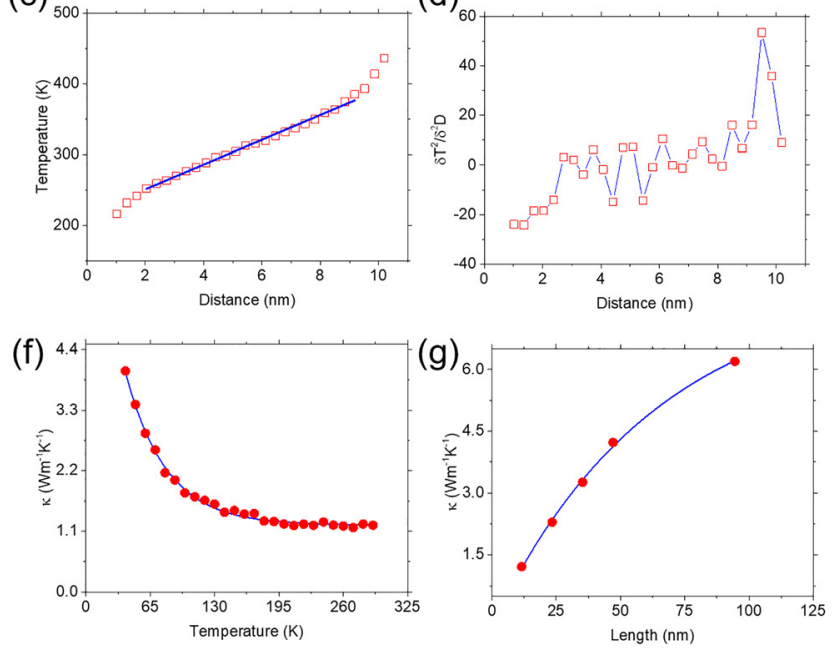

(b)

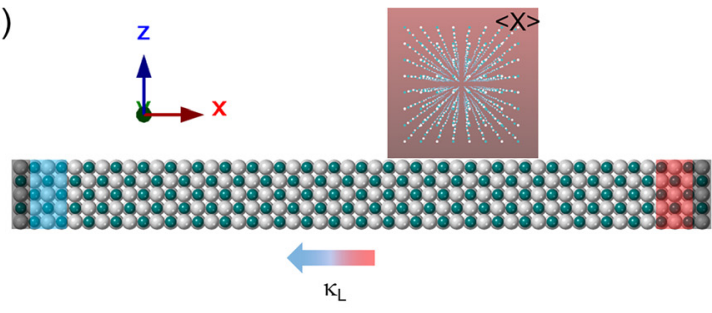

(e)
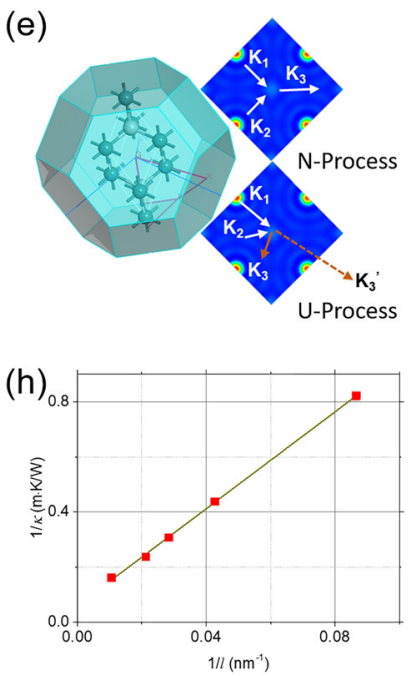

FIG. 3. (a) Left panel: standard cell of $\mathrm{PbS}$ shows a rock-salt cubic structure. Right panel: calculated phonon density-ofstates. (b) Atomic configurations used in the $\mathrm{PbS}$ nanowire MD simulations. The outermost atom layers denoted in black shades are fixed. The blue and red shaded areas stand for the heat sink and heat source, respectively. (c) Temperature distribution along the heat flux direction in the steady state. (d) Secondary-derivative length-dependent thermal distribution for estimating the length of the phonon free pathway, and $\mathrm{D}$ refers to the distance. (e) The first Brillouin zone for the primitive cell of $\mathrm{PbS}$ and two common phonon scattering processes. (f) Temperature dependence of thermal conductivity. (g) Dependence of thermal conductivity with the system length from 11.6 to $94.5 \mathrm{~nm}$. The blue dashed line is plotted for visual aid. (h) The inverse relationship of $1 / \kappa$ and 1/l. Linear fitting is employed to extrapolate the thermal conductivity of infinite length $\mathrm{PbS}$. 
[Fig. 3(f)]. Interestingly, we revealed that the predicted thermal conductivities showed a strong dependence on the system length $(l)$ when the system dimension is comparable to or smaller than the material intrinsic phonon-mean-free-path (MFP). ${ }^{32}$ In general, the thermal transport will become ballistic at small length scales when $l<$ MFP, and within the ballistic regime, certain phonon modes can transmit from the heatsource to the heat-sink without scattering. As shown in Figs. 3(g) and $3(\mathrm{~h})$, the calculated $\kappa$ increases from $1.2 \mathrm{~W} / \mathrm{m} \mathrm{K}$ to $6.2 \mathrm{~W} / \mathrm{m} \mathrm{K}$ when the $l$ value increases from $11.6 \mathrm{~nm}$ to $94.5 \mathrm{~nm}$ which is close to the predicted values of $4.14-4.29 \mathrm{~W} / \mathrm{m} \cdot \mathrm{K}$ from first principles calculations. ${ }^{33,34}$ This suggests that a low-dimensional nanostructure is generally beneficial for suppressing the thermal conductivity, but a less phonon quantum-confinement impact could be expected unless the dimension is reduced below $10 \mathrm{~nm}$. Therefore, we focus on improving the charge mobility through quantum-confinement while retaining the intrinsic low thermal conductivity to advance thermal transport modulation.

To understand the effect of QD shells for enhancing electronic transport, we evaluate the carrier mobility of the as-prepared p-NW@QDs through fabricating single-wire field-effect transistors (FETs). Figure 4(a) shows a scanning electron microscopy (SEM) image of the fabricated back-gated PbS NW FET with four electrodes (Fig. S7). The source-drain current-voltage $\left(\mathrm{I}_{\mathrm{ds}}-\mathrm{V}_{\mathrm{ds}}\right)$ plot [Fig. 4(b)] shows a linear-shaped (inset semilog plot) graph, which indicates that a Mott-type insulator to conductor transition occurred after the device fabrication. ${ }^{35}$ This may arise from the depletion of long-chain aliphatic ligands during the oxygen plasma and annealing process. ${ }^{36}$ In Fig. 4(c), the p-type characteristic of the asprepared $p$-NW@QD can be readily identified from the gate voltage and source-drain current $\left(\mathrm{V}_{\mathrm{g}}-\mathrm{I}_{\mathrm{ds}}\right)$ plots. P-Type nature is commonly observed in the solution-processed lead chalcogenide nanostructure, and its p-type characteristics are attributed to oxygen exposure and chemical interaction during device fabrication regardless of intrinsic stoichiometry. ${ }^{37}$ According to the cylinder-on-plate model, ${ }^{38}$ the maximum field-effect mobility was calculated to be $30.65 \mathrm{~cm}^{2} / \mathrm{Vs}$, which is much higher than bulk $\mathrm{PbS}\left(5 \mathrm{~cm}^{2} / \mathrm{Vs}\right)^{39}$ and other reported solution-processed analogous materials (Tables S1 and S2). ${ }^{2,40}$ We attribute this excellent hole mobility to the QD shells which boost the charge transport via manifold transfer dynamics (e.g., quantum tunneling or hopping). ${ }^{41}$ Indeed, various high mobility devices and our density functional theory (DFT) calculations (Figs. S8 and S9) revealed that long-range and fast charge transport could be realized in quantum confined nanostructures. ${ }^{42}$ In Figs. S8 and S9, through coating QDs on PbS nanowire surfaces, the calculated electron conductivity increased dramatically due to the improved tunneling charge transfer. The presence of QD allows electrons used to be free in three dimensions to be constrained in the orthogonal dimensions. While the dimension of the QD is comparable to the de Broglie wavelength of electrons, the generation of discrete energy values allows electrons to be able to tunnel through QDs. Also, the mobility value of PbS NW with a diameter of $46 \mathrm{~nm}$ $\left(26.6 \mathrm{~cm}^{2} / \mathrm{Vs}\right)$ showed no significant difference from that of $83 \mathrm{~nm}$ $\mathrm{PbS}$ (Fig. S10 and Table S1). This is because the mean-free-path of charge carriers in $\mathrm{PbS}$ is much shorter than the currently measured NW diameter. Therefore, the carrier transport mechanism within $p$ NW@QD can be rationalized as (i) the thermal lattice scattering is dominated by acoustic phonons in the wire cores; (ii) the electronic transport is mainly contributed from the surface QD shells. (a)

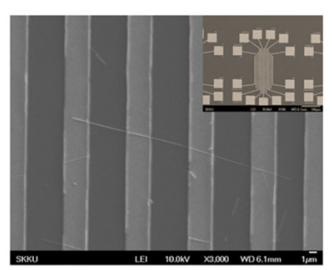

(b)

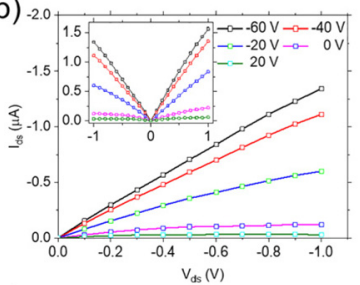

(c)

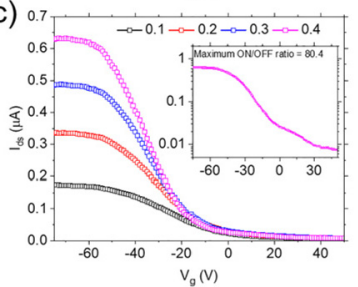

(d)

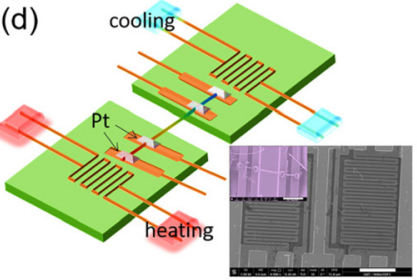

(e)

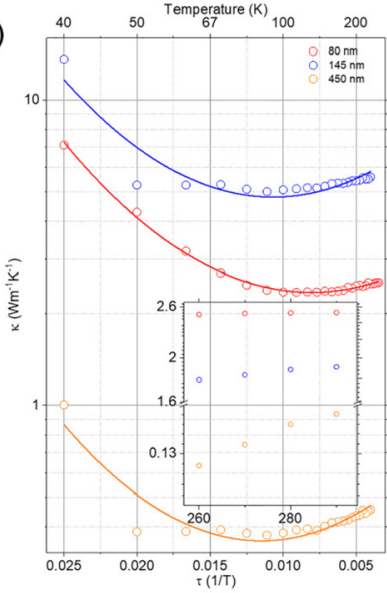

FIG. 4. (a) The SEM image of the fabricated back-gate FET. The inset shows the entire device morphology. The scale bars are 1 um and 100 um (inset), respectively. (b) Output $I_{d s}-V_{d s}$ curves with gate voltages from $-60 \mathrm{~V}$ to $20 \mathrm{~V}$ with a $20 \mathrm{~V}$ step. The inset shows the same plot on a log-scale. (c) Transfer $I_{d s}-V_{g}$ curves at different applied $V_{s d}$ conditions $(0.1 \mathrm{~V}-0.4 \mathrm{~V})$. The inset shows a log-scale $\mathrm{I}_{\mathrm{ds}}-\mathrm{V}_{\mathrm{g}}$ curve at $\mathrm{V}_{\mathrm{sd}}=0.4 \mathrm{~V}$ for determining the device ON-OFF ratio. (d) Top: Schematic illustration of the microdevice consisting of two suspended heater pads bridged by a PbS p-NW@QD. Bottom: Scanning electron microscopy (SEM) image of the microfabricated device for measuring thermal conductivity. The inset (high-resolution SEM) shows the detail configuration of the as-prepared microdevices. The scale bars are $10 \mathrm{um}$ and $3 \mathrm{um}$ (inset), respectively. (e) $\tau-\kappa$ Coordinate temperature dependence of thermal conductivity. The inset shows an enlarged graph within the temperature range of $250-300^{\circ} \mathrm{C}$.

To demonstrate the effect of the $p$-NW@QD core-shell structure on modulating thermal transport, we directly probed the heat flow kinetics through the state-of-the-art microfabricated suspended heatpad devices. The impacts of QD on the thermal conductivity have been theoretically perceived (Fig. S12) but have not been experimentally realized due to material preparation difficulties. ${ }^{7,16}$ As shown in Figs. 4(d), S13, and S14, drop-cast single PbS p-NW@QD was bridged on the device, and contact was made by finely depositing Pt through using a focused ion beam (FIB) system. ${ }^{43-45}$ Figures 4(e) and S15 show the thermal conductivity of the measured PbS p-NW@QD in the temperature range of $40 \mathrm{~K}-250 \mathrm{~K}$. The peak of thermal conductivity appeared below $50 \mathrm{~K}$ and the thermal conductivity plateaus located above $50 \mathrm{~K}$, which is relatively low compared to the previously reported silicacoated $\mathrm{PbS} \mathrm{NW} .^{40}$ This indicates that the phonon-phonon scattering is dominated by the U-process mechanism which is consistent with our conclusion derived from MD dynamical simulations. Moreover, as shown in the inset of Figs. 4(e) and S15, the as-prepared p-NW@QD does not exhibit a strong size-dependent effect in the current diameter range, which may be due to the fact that the PbS phonon mean-freepath is very short $(<10 \mathrm{~nm})$ and short-wavelength phonons can only 
be affected by point defects or alloy scattering rather than boundary scattering. Therefore, the thermal conductivity was determined through averaging the "single-wire-bridging" thermal flow as a function of their diameters $(\bar{k}=1.53 \pm 1 \mathrm{~W} / \mathrm{m} \mathrm{K})$, which manifests an overall smaller thermal conductivity compared to the bulk values (Table S2). ${ }^{10}$

In conclusion, we have prepared quantum dotlike nanostructure coated core-shell anisotropic NWs through a solvent-mediated approach. We exemplified the $p$-NW@QD structure via $\mathrm{PbS}$, which revealed that DER could be a sufficient coordinating reagent for tuning the morphology of NWs. Based on MD lattice dynamics simulations, we observed that the dimensional factor for efficient phonon scattering is limited below $10 \mathrm{~nm}$, and we confirmed that the U-process is the governing mechanism of the photon scattering behavior in the lowdimension thermal transport dynamics. Through fabricating fieldeffect transistors and microfabricated suspended heating devices, we demonstrated an anomalously high electronic mobility $\left(30.65 \mathrm{~cm}^{2} / \mathrm{Vs}\right)$ and low thermal conductivity $(1.53 \pm 1 \mathrm{~W} / \mathrm{m} \mathrm{K})$. Direct charge/heat carrier flow measurements and computational simulations confirm that the anomalous electrical and thermal transport phenomenon is strongly dependent on the fast charge transport through the QD shell, and a slow phonon migration across the Umklapp-process dominated NW cores. Our findings could provide theoretical and experimental guidance for the future synthesis and design of nanostructures for controlling the charge and heat transport in thermoelectric as well as topological insulator studies. The solution-processed nature also indicates that the growth of these $p$-NW@QD structures could be scaled up for mass production. We expect the material innovation and fast developments in the field of large-scale nanostructure fabrication to enable high-performance thermoelectric materials to be realized in the near future.

See the supplementary material for detailed experimental procedures and supporting images (Figs. S1-S15) and Tables S1 and S2.

We acknowledge funds from the Engineering and Physical Sciences Research Council (EPSRC, No. EP/P027628/1) and National Research Foundation of Korea grant funded by the Korea government (MSIT) (NRF, Nos. 2019R1A2C1005930 and 2017R1A2B2010663). Computational work was completed utilizing the Holland Computing Centre of the University of Nebraska, which receives support from the Nebraska Research Initiative of USA. B.H. would like to acknowledge EPSRC (No. EP/K040375/1) for funding the "South of England Analytical Electron Microscope" used in this research.

\section{REFERENCES}

'Z.-H. Ge, L.-D. Zhao, D. Wu, X. Liu, B.-P. Zhang, J.-F. Li, and J. He, Mater. Today 19(4), 227 (2016).

${ }^{2}$ O. Caballero-Calero and M. Martín-González, Scr. Mater. 111(Supplement C), 54 (2016)

${ }^{3}$ N. W. Ashcroft and N. D. Mermin, Solid State Physics (Holt, Rinehart and Winston, 1976).

${ }^{4}$ S. Yazdani and M. T. Pettes, Nanotechnology 29(43), 432001 (2018).

${ }^{5}$ S. Lee, K. Hippalgaonkar, F. Yang, J. Hong, C. Ko, J. Suh, K. Liu, K. Wang, J. J.

Urban, X. Zhang, C. Dames, S. A. Hartnoll, O. Delaire, and J. Wu, Science 355(6323), 371 (2017).

${ }^{6}$ A. Majumdar, Science 303(5659), 777 (2004).

${ }^{7}$ L. D. Hicks and M. S. Dresselhaus, Phys. Rev. B 47(19), 12727 (1993).
${ }^{8}$ J. Gooth, J. G. Gluschke, R. Zierold, M. Leijnse, H. Linke, and K. Nielsch, Semicond. Sci. Technol. 30, 015015 (2015).

${ }^{9}$ D. Wu, L.-D. Zhao, F. Zheng, L. Jin, M. G. Kanatzidis, and J. He, Adv. Mater. 28(14), 2737 (2016).

${ }^{10}$ H. Wang, E. Schechtel, Y. Pei, and G. J. Snyder, Adv. Energy Mater. 3(4), 488 (2013).

${ }^{11}$ A. L. Moore, S. K. Saha, R. S. Prasher, and L. Shi, Appl. Phys. Lett. 93(8), 083112 (2008).

${ }^{12}$ C. B. Vining, Nat. Mater. 8, 83 (2009).

${ }^{13}$ N. P. Dasgupta, J. Sun, C. Liu, S. Brittman, S. C. Andrews, J. Lim, H. Gao, R. Yan, and P. Yang, Adv. Mater. 26(14), 2137 (2014).

${ }^{14}$ P. M. Wu, J. Gooth, X. Zianni, S. F. Svensson, J. G. Gluschke, K. A. Dick, C. Thelander, K. Nielsch, and H. Linke, Nano Lett. 13(9), 4080 (2013).

${ }^{15}$ A. I. Hochbaum, R. Chen, R. D. Delgado, W. Liang, E. C. Garnett, M. Najarian, A. Majumdar, and P. Yang, Nature 451, 163 (2008).

${ }^{16}$ A. I. Boukai, Y. Bunimovich, J. Tahir-Kheli, J.-K. Yu, W. A. Goddard Iii, and J. R. Heath, Nature 451, 168 (2008).

${ }^{17}$ T. Markussen, Nano Lett. 12(9), 4698 (2012).

${ }^{18}$ F. W. Wise, Acc. Chem. Res. 33(11), 773 (2000).

${ }^{19}$ B. Hou, Y. Cho, B. S. Kim, J. Hong, J. B. Park, S. J. Ahn, J. I. Sohn, S. Cha, and J. M. Kim, ACS Energy Lett. 1, 834 (2016).

${ }^{20}$ B. Hou, Y. Cho, B.-S. Kim, D. Ahn, S. Lee, J. B. Park, Y.-W. Lee, J. Hong, H. Im, and S. M. Morris, J. Mater. Chem. C 5(15), 3692 (2017).

${ }^{21}$ Y. Tian, M. R. Sakr, J. M. Kinder, D. Liang, M. J. MacDonald, R. L. J. Qiu, H.-J. Gao, and X. P. A. Gao, Nano Lett. 12(12), 6492 (2012).

${ }^{22}$ S. Y. Jang, Y. M. Song, H. S. Kim, Y. J. Cho, Y. S. Seo, G. B. Jung, C.-W. Lee, J. Park, M. Jung, J. Kim, B. Kim, J.-G. Kim, and Y.-J. Kim, ACS Nano 4(4), 2391 (2010).

${ }^{23}$ K.-S. Cho, D. V. Talapin, W. Gaschler, and C. B. Murray, J. Am. Chem. Soc. 127(19), 7140 (2005).

${ }^{24}$ H. Wu, J. Carrete, Z. Zhang, Y. Qu, X. Shen, Z. Wang, L.-D. Zhao, and J. He, NPG Asia Mater. 6, e108 (2014).

${ }^{25}$ S. Johnsen, J. He, J. Androulakis, V. P. Dravid, I. Todorov, D. Y. Chung, and M. G. Kanatzidis, J. Am. Chem. Soc. 133(10), 3460 (2011).

${ }^{26}$ N. Shulumba, O. Hellman, and A. J. Minnich, Phys. Rev. B 95(1), 014302 (2017).

${ }^{27}$ P. Giannozzi, S. de Gironcoli, P. Pavone, and S. Baroni, Phys. Rev. B 43(9), 7231 (1991).

${ }^{28}$ Y. Yue, J. Zhang, Y. Xie, W. Chen, and X. Wang, Int. J. Heat Mass Transfer 110(Supplement C), 827 (2017).

${ }^{29}$ J. Zhang, Y. Wang, and X. Wang, Nanoscale 5(23), 11598 (2013).

${ }^{30} \mathrm{X}$. Wang, M. Wang, Y. Hong, Z. Wang, and J. Zhang, Phys. Chem. Chem. Phys. 19(35), 24240 (2017).

${ }^{31}$ C. Kittel, Introduction to Solid State Physics (Wiley, 1996).

${ }^{32}$ Y. Hong, J. Zhang, and X. Zeng, J. Phys. Chem. C 120(45), 26067-26075 (2016).

${ }^{33}$ Y. Zhang, X. Ke, C. Chen, J. Yang, and P. R. C. Kent, Phys. Rev. B 80(2), 024304 (2009).

${ }^{34}$ Z. Tian, J. Garg, K. Esfarjani, T. Shiga, J. Shiomi, and G. Chen, Phys. Rev. B 85(18), 184303 (2012).

${ }^{35}$ M. Law, J. M. Luther, Q. Song, B. K. Hughes, C. L. Perkins, and A. J. Nozik, J. Am. Chem. Soc. 130(18), 5974 (2008).

${ }^{36}$ J. Gao, S. Jeong, F. Lin, P. T. Erslev, O. E. Semonin, J. M. Luther, and M. C. Beard, Appl. Phys. Lett. 102(4), 043506 (2013).

${ }^{37}$ D. K. Kim, Y. Lai, T. R. Vemulkar, and C. R. Kagan, ACS Nano 5(12), 10074 (2011).

${ }^{38}$ Y. Cui, X. Duan, J. Hu, and C. M. Lieber, J. Phys. Chem. B 104(22), 5213 (2000).

${ }^{39}$ S. M. Salim and O. Hamid, Renewable Energy 24(3), 575 (2001).

${ }^{40} \mathrm{M}$. Fardy, A. I. Hochbaum, J. Goldberger, M. M. Zhang, and P. Yang, Adv. Mater. 19(19), 3047 (2007).

${ }^{41}$ C. R. Kagan, E. Lifshitz, E. H. Sargent, and D. V. Talapin, Science 353(6302) aac5523 (2016).

${ }^{42}$ H. Li, D. Zhitomirsky, S. Dave, and J. C. Grossman, ACS Nano 10(1), 606 (2016).

${ }^{43}$ D. Li, Y. Wu, P. Kim, L. Shi, P. Yang, and A. Majumdar, Appl. Phys. Lett. 83(14), 2934 (2003).

${ }^{44}$ E. K. Lee, L. Yin, Y. Lee, J. W. Lee, S. J. Lee, J. Lee, S. N. Cha, D. Whang, G. S. Hwang, K. Hippalgaonkar, A. Majumdar, C. Yu, B. L. Choi, J. M. Kim, and K. Kim, Nano Lett. 12(6), 2918 (2012).

${ }^{45}$ J. W. Lee, J. Lee, S.-H. Jung, Y. Jang, B. L. Choi, C.-W. Yang, D. Whang, and E. K. Lee, Nanotechnology 27(30), 305703 (2016). 